\title{
A STRONG EMISSION LINE NEAR 24.8 A IN THE X-RAY BINARY SYSTEM MAXI J0556-332: GRAVITATIONAL REDSHIFT OR UNUSUAL DONOR?
}

\author{
Dipankar Maitra $^{1}$, Jon M. Miller ${ }^{1}$, John C. Raymond ${ }^{2}$, And Mark T. Reynolds ${ }^{1}$ \\ ${ }^{1}$ Department of Astronomy, University of Michigan, Ann Arbor, MI 48109, USA; dmaitra@umich.edu \\ ${ }^{2}$ Harvard-Smithsonian Center for Astrophysics, 60 Garden Street, Cambridge, MA 02138, USA \\ Received 2011 July 22; accepted 2011 October 31; published 2011 November 18
}

\begin{abstract}
We report the discovery of a strong emission line near $24.8 \AA(0.5 \mathrm{keV})$ in the newly discovered X-ray binary system MAXI J0556-332 with the reflection grating spectrometer (RGS) on board the XMM-Newton observatory. The $\mathrm{X}$-ray light curve morphology during these observations is complex and shows occasional dipping behavior. Here we present time- and rate-selected spectra from the RGS and show that this strong emission line is unambiguously present in all the XMM observations. The measured line center is consistent with the Ly $\alpha$ transition of $\mathrm{N}$ VII in the rest frame. While the spectra contain imprints of absorption lines and edges, there appear to be no other significantly prominent narrow line due to the source itself, thus making the identification of the $24.8 \AA$ line uncertain. We discuss possible physical scenarios, including a gravitationally redshifted O VIII Ly $\alpha$ line originating at the surface of a neutron star or an unusual donor with an extremely high $\mathrm{N} / \mathrm{O}$ abundance $(>57)$ relative to solar that may have produced this comparatively strong emission line.
\end{abstract}

Key words: accretion, accretion disks - binaries: general - X-rays: binaries - X-rays: individual (MAXI J0556-332)

Online-only material: color figures

\section{INTRODUCTION}

The Monitor of All-sky X-ray Image (MAXI) detected a new $\mathrm{X}$-ray source on 2011 January 11, subsequently named MAXI J0556-332 (hereafter we will refer to the source as J0556; Matsumura et al. 2011). The discovery was immediately followed up by pointed observations in other wavelengths. X-ray observations made with Swift indicated that the interstellar extinction toward J0556 is small $\left(N_{\mathrm{H}} \sim 10^{21} \mathrm{~cm}^{-2}\right.$; Kennea et al. 2011) compared to most Galactic X-ray binaries (XRBs), which typically have $N_{\mathrm{H}} \sim 10^{22} \mathrm{~cm}^{-2}$. The low $N_{\mathrm{H}}$, largely owing to the high Galactic latitude of the source $(b=-25.183)$, implies that minimal line-of-sight absorption will facilitate efforts to study its low-energy X-ray spectrum. The X-ray spectral and timing properties of J0556 suggest that it is an XRB currently going through an episode of active accretion. The optical counterpart for this source was found to be a stellar object with USNO B1.0 magnitudes of $m_{R}=19.91$ and $m_{B}=19.52$, and an optical spectrum obtained by Halpern (2011) showed emission lines from $\mathrm{H} \alpha, \mathrm{He}$ I, and He II, indicating the presence of an accretion disk near the compact accretor. Space-borne X-ray and ground-based I-band monitoring (using the CTIO/SMARTS $1.3 \mathrm{~m}$ telescope) shows that the source is still active as of 2011 October 20.

The nature of the compact accretor or the companion donor star in J0556 is currently unknown, although most evidence point toward a neutron star (NS) accretor. No "type 1" bursts have been seen from the source so far, but the X-ray timing properties and evolution on the color-color diagram are similar to those of NS XRBs (Homan et al. 2011). The optical-to-X-ray and radio-to-X-ray flux ratios are also similar to Galactic NS XRBs (Russell et al. 2011; Coriat et al. 2011). The distance to the source is also currently unknown. Analyzing the archival data of the source from the Catalina Real-Time Transient Survey revealed that no optical outburst had been detected since 2005 August (Mahabal et al. 2011). Data from RXTE/All Sky Monitor show that this is its first X-ray outburst since 1996 January.

\section{DATA REDUCTION AND ANALYSIS}

XMM-Newton observed J0556 on MJD 55608 and 55653 (hereafter referred to as Obs1 and Obs2, respectively), each observation being of about $40 \mathrm{ks}$ duration. We analyzed the data obtained by the reflection grating spectrometer (RGS; den Herder et al. 2001) using the XMM-Newton Science Analysis Software (SAS; v.11.0.0) and following the standard extraction procedures outlined in the XMM-Newton User Guide. ${ }^{3}$ The background light curves were examined for the presence of proton flares. While there were no flares during Obs1, data from the initial $14 \mathrm{ks}$ of Obs2 had to be discarded due to high background. The SAS extraction regions for source and background spectra were inspected using the spatial- and energy-dispersion plots and left unchanged from the SAS default. The observed fluxes in each RGS CCD were compared with their 2\% pile-up flux limits (Section 3.4.4.8.1 of the $X M M$-Newton User's Handbook), and we found that pileup was significantly less than $2 \%$ even for the steady-high state (when the source was brightest among the two observations).

The average RGS count rates during Obs1 and Obs2 were 41.2 counts $\mathrm{s}^{-1}$ and 12.1 counts $\mathrm{s}^{-1}$, respectively. The light curve morphology during Obs1 (Figure 1) is quite complex, showing dipping behavior for $\sim 20 \mathrm{ks}$ during the middle of the observation as well as extended epochs without any dips. This confirms previous shorter X-ray observations of J0556 where the dips were seen occasionally (e.g., as reported by Strohmayer 2011; Belloni et al. 2011). Given the marked changes in the source behavior, it is likely that the spectral properties were changing during Obs1. Therefore, we extracted spectra from three separate intervals based on the combined RGS count rate (cps) and time ( $t$ seconds since the start of the observation): (1) steady-high-when $1000<t<11,000$ and cps $>45$, which constitutes most of the time when the source flux was steady;

\footnotetext{
3 http://xmm.esac.esa.int/external/xmm_user_support/documentation/ sas_usg/USG/
} 


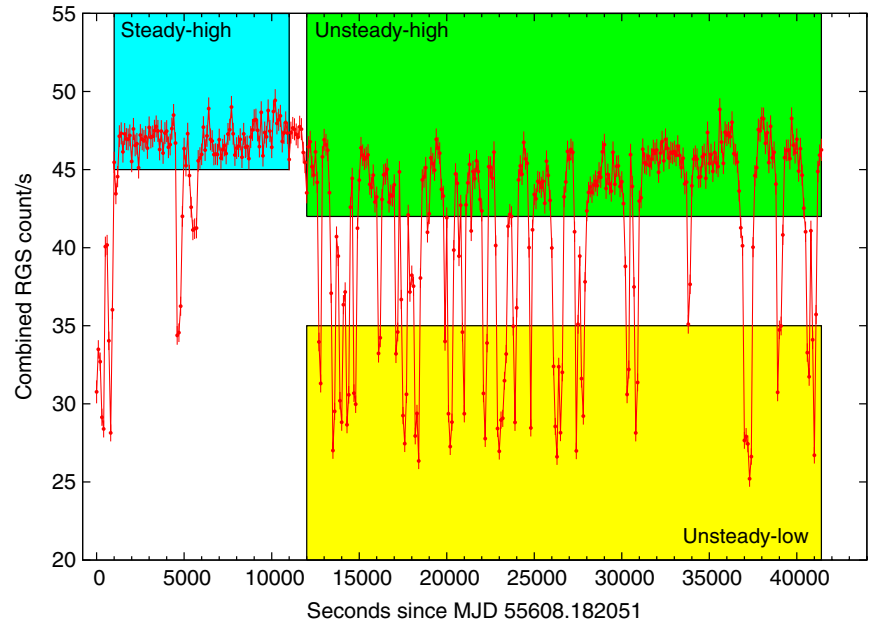

Figure 1. RGS light curve of the first XMM-Newton observation (Obs1) showing its complex morphology, especially epochs of dipping and non-dipping behavior. The variously colored regions represent the intervals from which spectra were analyzed.

(A color version of this figure is available in the online journal.)

(2) unsteady-high-when $t>12,000$ and cps $>42$, selecting the photons collected during the peaks of the dipping period; and (3) unsteady-low — when $t>12,000$ and cps $>35$, selecting the photons collected during the minima of the dipping period. These selections are marked in Figure 1 by differently colored regions. Once the background flare was excluded, the Obs2 light curve was steady and did not show any dips or any other signs of large variability. Therefore, we created one single time-averaged spectrum of the entire Obs2 after excluding the initial flare.

We analyzed data from the full RGS bandpass (7-36 $\AA$ ) for both RGS1 and RGS2 units. Since the second-order RGS spectra do not cover the wavelength region near the $24.8 \AA$ line, we restrict our analysis to the first-order spectra only. The spectra were grouped for all fits with the criterion that there were at least 30 counts bin $^{-1}$. Fits to the X-ray spectra were performed in wavelength space using ISIS (v.1.6.1-36; Houck \& Denicola 2000) and XSPEC (v12.7.0; Arnaud 1996). The solar abundance tables given by Anders \& Grevesse (1989) were used for all fits.

Apart from the $24.8 \AA$ emission line, which is readily visible in the RGS spectra (Figure 2), there are also many lowsignificance "bumps and wiggles." However, there appear to be no strong narrow spectral features associated with the source that are of comparable strength to the $24.8 \AA$ line. Signatures of the intervening interstellar medium (ISM) are seen via the presence of neutral and ionized atomic oxygen edges and absorption lines in the $\sim 22.1-23.8 \AA$ region. Also evident in the brighter observations is the instrumental neutral $\mathrm{O} \mathrm{K}$-edge absorption feature near $23.5 \AA$ as noted, e.g., by den Herder et al. (2001). There is a hint of an excess near $29 \AA$, which may be due to a blend of He-like N VI resonance line (28.78 $\AA$ in rest frame) and intercombination lines (29.082 and $29.084 \AA$ ). Note that the possible $\mathrm{N}$ vi feature does not show the forbidden component at $29.535 \AA$. But that is not surprising because the emission region in a compact XRB would be expected to be much denser than $10^{10} \mathrm{~cm}^{-3}$. Also, if the excess is indeed due to $\mathrm{N} \mathrm{VI}$, this would support a nitrogen-rich donor scenario. While there are no other narrow lines of comparable strength, there is a broad feature near $12.2 \AA(\sim 1 \mathrm{keV}$, covered only by RGS 2 and not by RGS 1$)$. The feature can be well modeled by a Gaussian centered near $12.2 \AA$ and a width of $1.4 \AA$. Such a broad feature near $\sim 1 \mathrm{keV}$ is sometimes seen in XRBs (see, e.g., Cackett et al. 2010). Given the quality of the data, we cannot rule out the possibility that it could be instrumental, or a relativistically broadened $\mathrm{Ne} x$ line, or a smeared Fe L-edge seen in reflection.

Various low-significance features are not only difficult to identify but also make the determination of the continuum difficult. For all the observations, the RGS continuum can be well modeled with a phenomenological broken power-law model. We also attempted to model the continuum with a thermal (both blackbody as well as accretion disk models were tried) plus power-law model. However, the thermal component in these models was not constrained and the best-fit temperatures were much higher than that of the energy range considered. Thus, in effect, the thermal+power-law models were mimicking a broken power law. The exact choice of continuum in the comparatively narrower RGS energy range is not crucial for measuring the $24.8 \AA$ line parameters, and we stress that the choice of the broken power-law model is nothing but an empirical description of the continuum for the purpose of measuring the line. A full analysis of the $0.6-10 \mathrm{keV}$ European Photon Imaging Camera (EPIC) data is beyond the scope and focus of the current Letter, and will be presented in a later work (D. Maitra et al. 2012, in preparation). However, we would briefly like to note that the phenomenological thermal+power-law fits to the EPIC pn data shows no strong evidence for hardening of the power-law component during the dips. The power-law photon index during dips (unsteady-low) is $\Gamma=2.65 \pm 0.10$ and that outside dips (unsteady-high) is $2.51 \pm 0.06$. However, both the temperature and normalization of the thermal component are smaller during the dips (when $k T_{\mathrm{BB}}=0.70 \pm 0.15 \mathrm{keV}, N_{\mathrm{BB}}=$ $90 \pm 21)$ when compared to those outside the dips $\left(k T_{\mathrm{BB}}=\right.$ $0.81 \pm 0.02 \mathrm{keV}, N_{\mathrm{BB}}=143 \pm 13$ ). As a result, the ratio of unabsorbed non-thermal-to-thermal flux within the EPIC pn energy range is higher during the dips compared to that outside the dips. We modeled absorption of X-rays by the ISM using the Tuebingen-Boulder model (tbnew; an updated version of Wilms et al. 2000). The best-fit model parameters for the various observations/selections are given in Tables 1 and 2. The quoted errors for the best-fit models correspond to a $90 \%$ confidence limit for the continuum model parameters and $68 \%$ confidence limit for line parameters.

While fitting, the normalization of RGS2 was allowed to vary w.r.t. that of RGS1. The fits show that the RGS2 normalization factor is in the range of 0.94-0.97. Figure 3 shows the $24.8 \AA$ line flux and 26-28 $\AA$ continuum flux for the various observations/ selections. Note that the ratio of line-to-continuum flux during Obs2 $(0.11 \pm 0.03)$ is similar to that during the steady-high state of Obs1 $(0.14 \pm 0.02)$. This strongly suggests that the line and the continuum fluxes are causally linked and both originate from the accreting system (therefore ruling out the possibility that the line is from some other astronomical source in the angular proximity of J0556; furthermore, there is no other source of comparable X-ray brightness in the EPIC image).

\section{ABUNDANCE ESTIMATES USING XSTAR PHOTOIONIZATION CODE}

In order to obtain a self-consistent understanding of the elemental abundances in the observed spectra we used the XSTAR photoionization code (Kallman \& Bautista 2001). A grid of 480 XSTAR models was created from the following parameters: (1) model column density of the emitting plasma was sampled between $10^{19}$ and $10^{24} \mathrm{~cm}^{-2}$, (2) the ionization parameter $\left(\log (\xi)\right.$ where $\left.\xi=L / n R^{2}\right)$ was sampled between 0 and 4, (3) $\mathrm{N}$ abundance was sampled between 1 and 100 times 


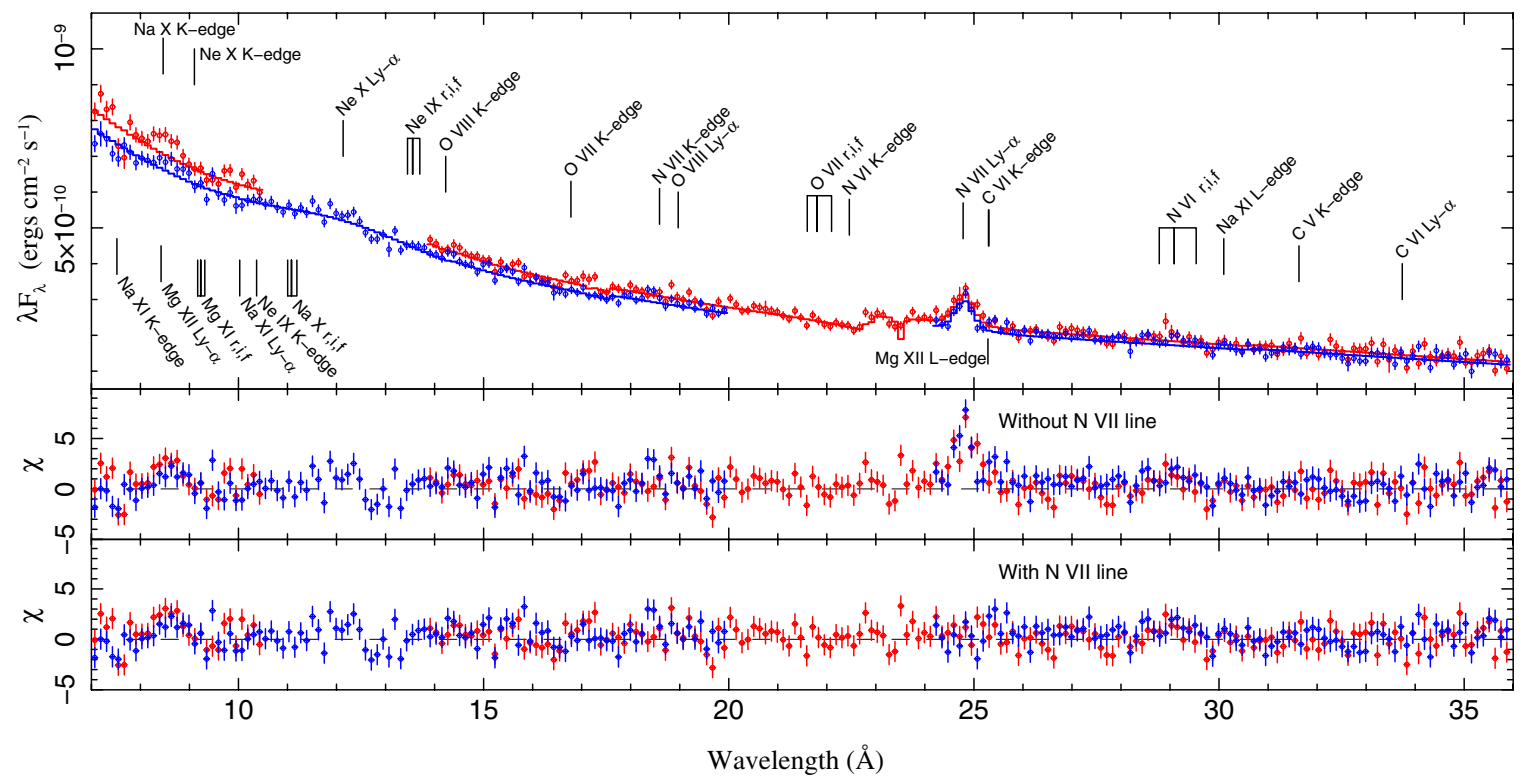

Figure 2. XMM-Newton/RGS spectrum of J0556, in 7-36 ̊ range, obtained on MJD 55608 during the steady-high period (i.e., the cyan region in Figure 1). Top panel: RGS1 data in red and the RGS2 data in blue (binned for visual clarity only). Data from both RGS units were fitted jointly as described in text. The joint best-fit models for RGS1 and RGS2 are shown by red and blue histograms, respectively. Rest-frame energies of few different lines/edges typically prominent in this range are labeled. The energies of the resonance (r), intercombination (i), and forbidden (f) lines are also shown. The broad feature in the 10-14 A range was modeled with a Gaussian centered at $12.2 \AA$ and width $(\sigma)$ of $1.4 \AA$. Middle panel: residuals in units of standard deviation, when the normalization of the narrow line at $24.8 \AA$ $(0.5 \mathrm{keV})$ was set to zero. Bottom panel: residuals in units of standard deviation, to the best-fit continuum + line model.

(A color version of this figure is available in the online journal.)

Table 1

Best-fit Continuum Parameters for the RGS Data ${ }^{a}$

\begin{tabular}{lccccr}
\hline \hline Obs/Selection & $\begin{array}{c}N_{\mathrm{H}} \times 10^{20} \\
\left(\mathrm{~cm}^{-2}\right)\end{array}$ & $\Gamma_{1}$ & $\begin{array}{c}E_{\mathrm{b}} \\
(\mathrm{keV})\end{array}$ & $\Gamma_{2}$ & $N$ \\
\hline Obs1/steady-high & $3.88_{-0.74}^{+0.71}$ & $1.87_{-0.28}^{+0.28}$ & $0.475_{-0.012}^{+0.019}$ & $1.20_{-0.05}^{+0.05}$ & $0.203_{-0.031}^{+0.035}$ \\
Obs1/unsteady-high & $2.29_{-0.25}^{+0.25}$ & $1.18_{-0.03}^{+0.03}$ & $1.491_{-0.048}^{+0.050}$ & $1.60_{-0.15}^{+0.18}$ & $0.303_{-0.003}^{+0.003}$ \\
Obs1/unsteady-low & $3.57_{-0.65}^{+0.18}$ & $2.58_{-0.98}^{+0.42}$ & $0.396_{-0.014}^{+0.022}$ & $1.57_{-0.05}^{+0.05}$ & $0.079_{-0.028}^{+0.113}$ \\
Obs2 & $3.33_{-0.46}^{+0.73}$ & $2.09_{-0.56}^{+0.91}$ & $0.396_{-0.030}^{+0.050}$ & $1.31_{-0.04}^{+0.05}$ & $0.041_{-0.026}^{+0.028}$ \\
\hline
\end{tabular}

Note. ${ }^{a}$ Errors are $90 \%$ confidence limits. Column (1) gives the observation and time/rate selection as discussed in Section 2; Column (2) is the best-fit $N_{\mathrm{H}}$ in units of $10^{20}$ atoms $\mathrm{cm}^{2}$; Columns (3) and (5) are power-law photon indices for $E<E_{b}$ and $E>E_{b}$, respectively; Column (4) is the break point for the energy; Column (6) is the power-law normalization at $1 \mathrm{keV}$, i.e., photons $\mathrm{keV}^{-1} \mathrm{~cm}^{-2} \mathrm{~s}^{-1}$ at $1 \mathrm{keV}$.

Table 2

Best-fit Narrow Line Parameters for the RGS Data ${ }^{a}$

\begin{tabular}{lccccr}
\hline \hline Obs/Selection & $\begin{array}{c}\text { Center } \\
(\AA)\end{array}$ & $\begin{array}{c}\sigma \\
(\AA)\end{array}$ & $\begin{array}{c}10^{3} \times \text { Flux } \\
(\text { photons cm }\end{array}$ & $\begin{array}{c}\text { Equiv. width } \\
(\mathrm{m} \AA)\end{array}$ & $\chi^{2} / \nu$ \\
\hline Obs1/steady-high & $24.801_{-0.017}^{+0.018}$ & $0.168_{-0.018}^{+0.020}$ & $2.96_{-0.23}^{+0.23}$ & $190_{-15}^{+15}$ & $4650.2 / 3609$ \\
Obs1/unsteady-high & $24.800_{-0.014}^{+0.014}$ & $0.211_{-0.022}^{+0.026}$ & $3.78_{-0.21}^{+0.22}$ & $273_{-15}^{+16}$ & $5138.0 / 3902$ \\
Obs1/unsteady-low & $24.824_{-0.016}^{+0.016}$ & $0.175_{-0.023}^{+0.026}$ & $4.13_{-0.30}^{+0.31}$ & $343_{-25}^{+26}$ & $2632.1 / 2575$ \\
Obs2 & $24.738_{-0.029}^{+0.029}$ & $0.219_{-0.038}^{+0.053}$ & $0.66_{-0.07}^{+0.08}$ & $155_{-17}^{+18}$ & $4213.0 / 3580$ \\
\hline
\end{tabular}

Note. ${ }^{a}$ Errors are $68 \%$ confidence limits. Column (1) same as in Table 1; Columns (2)-(5) best-fit Gaussian line center, width $(\sigma)$, line flux, and equivalent width for the narrow line; Column (6) $\chi^{2} /$ dof for the best fit to the 7-36 ̊ RGS data.

relative to solar, and (4) $\mathrm{O}$ abundance sampled between 0.01 and 1 times relative to solar. A hydrogen nucleus density of $10^{12} \mathrm{~cm}^{-3}$ and a covering fraction of 0.5 were assumed. A high turbulent velocity of $1000 \mathrm{~km} \mathrm{~s}^{-1}$ was used to simulate the line broadening. Phenomenological blackbody+power-law fits to the $0.6-10 \mathrm{keV}$ EPIC pn data obtained during the Obs1/steady-high state $\left(k T_{\mathrm{BB}}=0.86 \mathrm{keV}, N_{\mathrm{BB}}=147, \Gamma_{\mathrm{PL}}=\right.$ $2.20, N_{\mathrm{PL}}=0.42$ ) were used as user-defined incident radiation field in XSTAR. For a source distance of $1 \mathrm{kpc}$ (note that Welsh et al. 2010 reported a cloud of $\mathrm{Na}$ I and Ca II at $\sim 100 \mathrm{pc}$ in the direction of J0556; moreover, given the high galactic longitude of J0556, a distance of $\gg 1 \mathrm{kpc}$ would put the source far out of the galactic plane), the flux measured by EPIC pn implies a luminosity of $2.6 \times 10^{35} \mathrm{erg} \mathrm{s}^{-1}$. The remaining XSTAR parameters were set to their default values. An XSPEC table model created from this grid was fit to Obs1/steady-high state. 


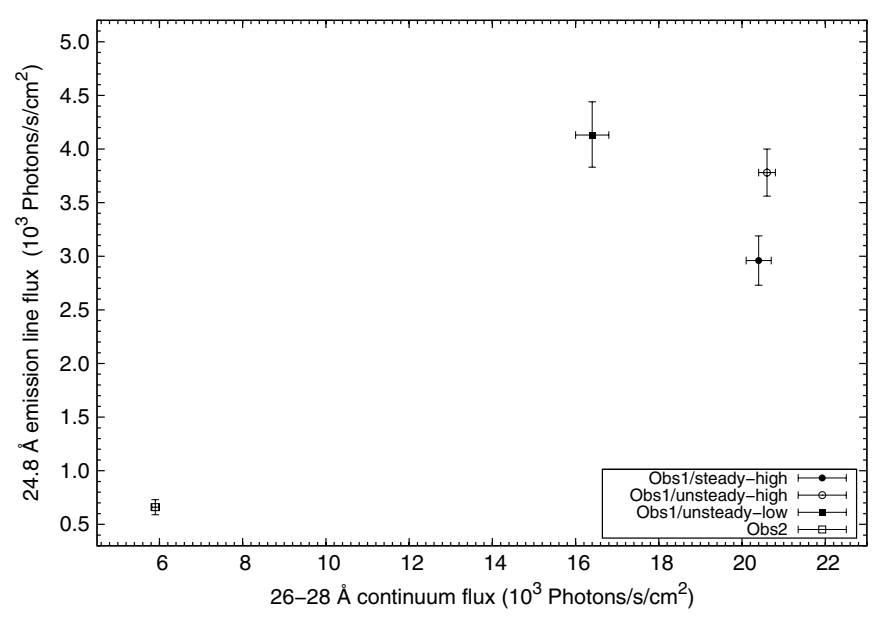

Figure 3. Photon flux from the $24.8 \AA$ emission line vs. $26-28 \AA$ continuum flux during the various observations/selections.

The best fit suggests a model column density of $3.2 \times 10^{21} \mathrm{~cm}^{-2}$ and $\log (\xi)=2.3$. The $90 \%$ confidence lower $\operatorname{limit}$ on $\log (\xi)$ is 2.1 (mainly based on an upper limit on the $\mathrm{N}$ VI/N viI ratio), but the upper limit on $\log (\xi)$ could not be constrained from the data. Since there is only one statistically significant narrow line in the spectrum, it is not possible to constrain the abundance of any single element. Rather, the fits are only able to give limits on the relative $\mathrm{N} / \mathrm{O}$ ratio. The fits suggest $\mathrm{N} / \mathrm{O}$ overabundance $>57$ ( $>27$ at $90 \%$ confidence) with respect to solar. This translates to an absolute $\mathrm{N} / \mathrm{O}$ of $>7.5$ ( $>3.6$ at $90 \%$ confidence) since we use the solar abundance table of Anders \& Grevesse (1989).

We also created XSTAR models assuming the line is due to redshifted $\mathrm{O}$ VIII Ly $\alpha$ and solar nitrogen abundance. The best fit to this redshifted O vIII model with $\chi^{2} / v=4702 / 3608$ is statistically slightly less favored than the N-rich model with $\chi^{2} / v=4673 / 3607$. We note that the best-fit $\log (\xi)$ was pegged at the maximum value allowed by our redshifted oxygen table model, implying a rather high $\log (\xi)>6$ would be needed in that scenario.

\section{DISCUSSION AND CONCLUSIONS}

J0556 is one of the few exceptional XRBs whose low-energy $\mathrm{X}$-ray spectrum can be studied in detail due to low interstellar extinction. Fits to its RGS spectra imply a column density of $(2.1-4.6) \times 10^{20} \mathrm{~cm}^{-2}$, which is at least an order of magnitude below most XRBs. Both XMM-Newton observations of J0556 show a strong emission line near $24.8 \AA$. If the line is from $\mathrm{N}$ VII, this would require the donor to have an extremely high $\mathrm{N} / \mathrm{O}$ abundance $(>57)$ relative to solar, based on the weakness of oxygen lines of similar charge states. Typically, for an XRB with a solar-type donor, the oxygen abundance is $\sim 8 \times$ greater than that of nitrogen, and the X-ray spectra show lines from $\mathrm{H}$ - and He-like ions of oxygen. Even in the intermediate-mass XRB Her X-1, wherein N/O is $4 \times$ solar, O lines are observed to be as strong or much stronger than $\mathrm{N}$ lines from similar charge states (see, e.g., Jimenez-Garate et al. 2002). While $\mathrm{N}$ overabundance is not uncommon in low-mass XRBs (e.g., in four of five UV spectra analyzed by Raymond 1993 the N/C ratios were several times solar, with a 9:1 ratio in Cyg $\mathrm{X}-2$ being highest), the extremely high N/O in J0556 makes it a unique XRB.

Given the lack of source distance and any signature of orbital periodicity so far, it is only possible to speculate on the nature of the donor star in this system based on its strong $\mathrm{N} / \mathrm{O}$ overabundance and color (see below), and exotic stars such as hot, core-helium-burning subdwarfs ( $\mathrm{sdB}$, sdO), or degenerate white dwarfs (WD) appear to be strong candidates. Hot subdwarfs were first discovered at high Galactic latitudes by Humason \& Zwicky (1947), and subsequent studies have shown that about half of them are in binary systems with WD or low-mass main-sequence stars. It is generally thought that the hydrogen-rich outer envelope of the progenitor hot subdwarf in a binary system is lost via either Roche lobe overflow or commonenvelope ejection mechanism, thus exposing CNO products dredged up from the core (see, e.g., Han et al. 2002). Naslim et al. (2010) have recently determined abundances for six sdB stars. The absolute $\mathrm{N} / \mathrm{O}$ ratios were 10,13 , and 20 for three of them (SB 21, LB 1766, and BPS CS 22940-0009), and oxygen was not detected in the other three. These stars were Ne-rich as well, so future observations of the Ne spectrum of J0556 would be especially interesting. One particular sdB star, PG $1219+534$, is observed to have N/O $\gtrsim 100$ (see, e.g., Heber 2009 for a review). Based on recent evolutionary calculations studying formation of ultracompact binaries (UCBs) by Nelemans et al. (2010), it is also possible that the donor is a helium WD. In the absence of detection of any $\mathrm{C}$ lines, $\mathrm{N} / \mathrm{C}$ ratio is unconstrained but likely high given the strength of the $\mathrm{N}$ VII line. Based on the diagnostics given by Nelemans et al. (2010), a high N/C would also point toward a helium WD.

Assuming that the magnitudes reported in the USNO-B1.0 catalog for the optical counterpart were made during quiescence, the temperature inferred from the de-reddened $B-R$ color is $\sim 46,000 \mathrm{~K}$. This almost certainly rules out typical donors found in low-mass XRBs, which tend to be of late spectral type ( $\mathrm{K}$ and $\mathrm{M})$ and hence cooler. The high temperature suggested by the USNO photometry would also support a hot subdwarf donor scenario. A caveat to the above scenario is that the mass transfer rate during quiescence may also be non-negligible, so that there exists an accretion disk even during X-ray quiescence (making the optical colors appear blue). Also, He-sdOs are thought to evolve from mergers of WDs, and it may be difficult to place such a merger product in a binary with an NS. The low column density toward the source makes it unlikely that the donor is a Wolf-Rayet star because Wolf-Rayet stars drive massive winds creating high internal columns, e.g., as seen in Cyg X-3.

If the donor is indeed a $\mathrm{WD} / \mathrm{sdB} / \mathrm{sdO}$, then the system is likely a UCB with orbital period ranging from a few tens of minutes to $\sim$ few hours. Observations of such UCBs are sparse, and their evolution is only recently being explored thoroughly (see, e.g., Tauris \& van den Heuvel 2006; Nelemans et al. 2010). From a sample of $50 \mathrm{sdB}$ binaries, Geier et al. (2008) found that possibly four could have an NS or black hole accretor. However, despite a sensitive search, no radio pulsations have yet been detected from these four systems (Coenen et al. 2011). If the donor in J0556 is a hot subdwarf, this would be the first direct evidence of a binary system with a hot subdwarf donor and a compact (black hole or NS) accretor.

Another intriguing possibility, assuming solar-abundance plasma, is that the observed line is a gravitationally redshifted O vIII Ly $\alpha$ line (rest frame $\lambda=18.967 \AA$ ) originating from the surface of the NS. A photon emitted with a rest-frame energy of $E_{0}$ from the surface of a slowly rotating, spherically symmetric NS of mass $M_{\mathrm{NS}}$ and radius $R_{\mathrm{NS}}$ is observed by an observer at infinity to have an energy $E$, where $E / E_{0}=$ $\sqrt{1-\left(2 G / c^{2}\right)\left(M_{\mathrm{NS}} / R_{\mathrm{NS}}\right)}$. Based on the most precise mass estimates from pulsar timing experiments, observed NS masses 
range between 1.25 and $2 M_{\odot}$ (see, e.g., Kramer \& Wex 2009; Demorest et al. 2010). Assuming the observed $24.8 \AA$ line in $\mathrm{J} 0556$ is gravitationally redshifted $\mathrm{O}$ VIII $\mathrm{Ly} \alpha$, the above mass range would imply a radius of $8.9-14.2 \mathrm{~km}$. In this redshifted oxygen line scenario, the width of the line could be used to put an upper limit on the relative size of the emission region as follows: assume that the width of the observed line profile is mainly due to superposition of lines originating at various heights above the NS. Since a photon emitted from the surface (i.e., from a radius of $R_{\mathrm{NS}}$ ) is more redshifted than a photon emitted from $R_{\mathrm{NS}}+\Delta R$, the observed width of the narrow line may be related to $\Delta R$ as $\Delta R / R_{\mathrm{NS}} \sim \sigma /\left(E_{0}-E\right) \sim 0.023$. This is obviously an upper limit to $\Delta R$, since it ignores any bulk motion. However, a redshifted $\mathrm{O}$ VIII line scenario would also require other redshifted lines of oxygen to be present in the spectra, which are not seen. Also, the hint of N vi resonance and intercombination lines favors the N/O overabundance scenario. Planned high-resolution optical/UV spectroscopy will be key in understanding the nature of this unique source.

We thank the anonymous referee for helpful comments. It is a pleasure to thank Norbert Schartel and the XMM-Newton planning team for carrying out the ToO observations. We acknowledge the use of the daily monitoring data obtained by the RXTE which has provided excellent all-sky coverage of $\mathrm{X}$-ray sources over the past 15 years.

\section{REFERENCES}

Anders, E., \& Grevesse, N. 1989, Geochim. Cosmochim. Acta, 53, 197 Arnaud, K. A. 1996, in ASP Conf. Ser. 101, Astronomical Data Analysis Software and Systems V, ed. G. H. Jacoby \& J. Barnes (San Francisco, CA: ASP), 17
Belloni, T., Motta, S., Munoz-Darias, T., \& Stiele, H. 2011, ATel, 3112, 1

Cackett, E. M., Miller, J. M., Ballantyne, D. R., et al. 2010, ApJ, 720, 205

Coenen, T., van Leeuwen, J., \& Stairs, I. H. 2011, A\&A, 531, A125

Coriat, M., Tzioumis, T., Corbel, S., et al. 2011, ATel, 3119, 1

Demorest, P. B., Pennucci, T., Ransom, S. M., Roberts, M. S. E., \& Hessels, J. W. T. 2010, Nature, 467, 1081

den Herder, J. W., Brinkman, A. C., Kahn, S. M., et al. 2001, A\&A, 365, L7

Geier, S., Karl, C., Edelmann, H., Heber, U., \& Napiwotzki, R. 2008, in ASP Conf. Ser. 392, Hot Subdwarf Stars and Related Objects, ed. U. Heber, C. S. Jeffery, \& R. Napiwotzki (San Francisco, CA: ASP), 207

Halpern, J. P. 2011, ATel, 3104, 1

Han, Z., Podsiadlowski, P., Maxted, P. F. L., Marsh, T. R., \& Ivanova, N. 2002, MNRAS, 336, 449

Heber, U. 2009, ARA\&A, 47, 211

Homan, J., Linares, M., van den Berg, M., \& Fridriksson, J. 2011, ATel, 3650, 1

Houck, J. C., \& Denicola, L. A. 2000, in ASP Conf. Ser. 216, Astronomical Data Analysis Software and Systems IX, ed. N. Manset, C. Veillet, \& D. Crabtree (San Francisco, CA: ASP), 591

Humason, M. L., \& Zwicky, F. 1947, ApJ, 105, 85

Jimenez-Garate, M. A., Hailey, C. J., den Herder, J. W., Zane, S., \& Ramsay, G. 2002, ApJ, 578, 391

Kallman, T., \& Bautista, M. 2001, ApJS, 133, 221

Kennea, J. A., Evans, P. A., Krimm, H., et al. 2011, ATel, 3103, 1

Kramer, M., \& Wex, N. 2009, Class. Quantum Gravity, 26, 073001

Mahabal, A. A., Drake, A. J., Djorgovski, S. G., et al. 2011, ATel, 3328, 1

Matsumura, T., Negoro, H., Suwa, F., et al. 2011, ATel, 3102, 1

Naslim, N., Jeffery, C. S., Ahmad, A., Behara, N. T., \& Şahìn, T. 2010, MNRAS, 409,582

Nelemans, G., Yungelson, L. R., van der Sluys, M. V., \& Tout, C. A. 2010, MNRAS, 401, 1347

Raymond, J. C. 1993, ApJ, 412, 267

Russell, D. M., Lewis, F., Doran, R., \& Roberts, S. 2011, ATel, 3116, 1

Strohmayer, T. E. 2011, ATel, 3110, 1

Tauris, T. M., \& van den Heuvel, E. P. J. 2006, in Compact stellar X-ray sources, ed. W. Lewin \& M. van der Klis (Cambridge: Cambridge Univ. Press), 623

Welsh, B. Y., Lallement, R., Vergely, J.-L., \& Raimond, S. 2010, A\&A, 510, A54

Wilms, J., Allen, A., \& McCray, R. 2000, ApJ, 542, 914 